\title{
Automated surface inspection using Gabor filters
}

\author{
D. M. Tsai and S. K. Wu \\ Machine Vision Lab. \\ Department of Industrial Engineering and Management \\ Yuan-Ze University, Chung-Li, Taiwan, R.O.C. \\ E-mail: iedmtsai@saturn.yzu.edu.tw
}

\begin{abstract}
In this paper we present a machine vision system for automatic inspection of defects in textured surfaces found in industry. The defects to be inspected are those that appear as local anomalies embedded in a homogeneous texture. The proposed method is based on a Gabor filtering scheme that computes the output response of energy from the convolution of a textured image with a specific Gabor filter. The best parameters of a Gabor filter is selected so that the energy of the homogeneous texture is zero, and any unpredictable defects will generate significantly large energy values. A simple thresholding scheme then follows to discriminate between homogeneous regions and defective regions in the filtered image. This transforms texture differences into detectable filter output. The experiments on structural textures such as textile fabrics and milled surfaces, and statistical textures such as leather and sandpaper have shown the effectiveness of the proposed method.
\end{abstract}

Key words: Machine vision, Surface inspection, Defect detection, Gabor filter 


\section{INTRODUCTION}

Image analysis techniques are being increasingly used to automate industrial inspection. The manual activity of inspection could be subjective and highly dependent on the experience of human inspectors. In this study we use machine vision to substitute for human inspectors for automated surface inspection.

In automated surface inspection, one has to solve the problem of detecting small surface defects which appear as local anomalies embedded in a homogeneous texture. Textures are generally classified into two major types, structural and statistical [1]. Structural textures are those that are composed of repetitions of some basic texture primitives with a deterministic rule of displacement. This type of defect inspection arises in textile fabrics and machined surfaces. Statistical textures can not be described by primitives and displacement rules. The spatial distribution of gray levels in such textured image is rather stochastic. The textured surfaces of sandpaper and castings fall in this category. In this paper, we aim at the surface defect inspection for both structural and statistical textures found in industry.

The inspection task in this paper is classified as qualitative inspection [2] which involves detecting non-quantitatively measurable but obviously faulty items such as scratches, cracks, stains, shedding of fibers and other ill-defined faults. Texture defects may have block-like or linear shapes or any random patterns. The sizes of defects are usually small with respect to the imaged surface, which break the homogeneity of the texture pattern. Human inspectors are quite flexible and can easily recognize novel defects in textured surfaces. Many of these unanticipated 
defects can not be described by explicit measures, making automated defect detection difficult.

Most of the defect detection systems are focused on nontextured surfaces (uniform gray-level images), such as glass panel [3], sheet steel [4], aluminum strips [5], and web materials [6], using thresholding or edge detection techniques. Defects in these images can easily be detected because commonly used measures usually have very distinct values. All automated visual inspection systems for complicated textured-surfaces generally attempt to identify defects by building adequate representation (templates or features) for the model image and using this representation to analyze a sensed image for detecting anomalies. The degree of success of an inspection system depends on how adequate and general the representation is. Automated visual inspection techniques can be separated into two general approaches based on the representation used [2]. The first approach involves matching a template of a defect-free model to the sensed image. This pixel-by-pixel matching scheme has been widely used for PCB (printed circuit board) inspection [7, 8]. However, it is only suitable for objects with geometric patterns. It experiences difficulty with frequently occurring variations of textured patterns.

The second approach computes a set of textural features in a sliding window (subimage), and searches for significant local deviations in the feature values from the entire image. The most difficult task of this approach is to extract adequate textural features which most completely embody information of the texture in the image. The features generally must be evaluated from samples of a large training set. A set of features that is an optimal representation of a specific texture could be completely 
useless for other texture patterns. There is no straightforward manner to judge the appropriate features to use. Therefore, the selection of an adequate feature set for a new texture in the training process requires the help of human knowledge. Furthermore, textures which are characterized by a vector of multiple features result in high dimensionality. This call for complicated classifiers such as Bayes [9], maximum likelihood [10], and neural networks [11] for comparing sensed features and model features.

Many methods have been proposed to extract textural features either directly from the spatial domain or from the spatial-frequency domain. In the spatial domain, the more simple textural features are first-order statistics [12] such as mean, variance, skewness and kurtosis from the gray-level histogram of an image. The more reliable and commonly used features are the second-order statistics derived from spatial gray-level co-occurrence matrices [13]. A co-occurrence matrix is based on the estimation of a second-order joint conditional probability density function that defines the spatial and intensity changes between pixels. A series of statistics, such as energy, entropy, local homogeneity, etc, is then calculated from the co-occurrence matrix as the discriminate measures of textures. A survey and demonstration of the co-occurrence matrix methods can be found in [14].

Ojala et al. [15] used textural features based on first-order statistics derived from edges in the image, and Conners et al. [16] employed both first-order statistics and second-order statistics derived from co-occurrence matrices for the inspection of defects in wood. Siew and Hogdson [14] studied textural features for carpet wear assessment based on co-occurrence matrices. Ramana and Ramamoorthy [12] used 
the co-occurrence matrix approach to study the textural features of machined surfaces in grinding, milling and shaping processes.

Early work [17] suggested that it may be possible to find better textural features, which are less sensitive to noise and intensity variation, in the spatial-frequency domain than those features extracted from the spatial domain. Measures of the shape of the spatial frequency spectrum such as location, size and orientation of peak in regions of spatial frequency using the 2-D Fourier transform can be used to design the textural features. Liu and Jernigan [18] presented a set of 28 textural features derived in the spatial frequency domain for texture analysis. Tsai et al. [19] proposed 5 textural features derived from the Fourier domain image and used neural network classifiers to assess the roughness of machined surfaces.

The Fourier methods characterize the spatial-frequency distribution, but they do not consider the information in the spatial domain. In the recent past, Gabor filters [20] are well recognized as a joint This type of defect inspection arises in textile fabrics and machined surfaces. spatial/spatial-frequency representation for analyzing textured images containing highly specific frequency and orientation characteristics. Daugman [21] showed that Gabor filters have optimal joint localization in both the spatial and the spatial-frequency domains. In addition, they are bandpass filters, which are inspired by a multi-channel filtering theory for processing visual information in the early stages of the human visual system [22, 23].

A 2-D Gabor function is an oriented complex sinusoidal grating modulated by a 2-D Gaussian function. The parameters of the Gabor function are specified by the 
frequency, the orientation of the sinusoid, and the scale of the Gaussian function. Local orientations and spatial frequencies explicit in Gabor filters are therefore used as the key features for texture processing. Gabor-filter based methods have been successfully applied for texture segmentation [24-29], where the input image is generally filtered by a family of Gabor filters tuned to several resolutions and orientations. However, it may not be computationally convenient or feasible to apply a large number of filters representing a variety of defect patterns.

This paper considers the issue of designing a single Gabor filter to detect any unpredictable defects in a specific textured-surface. The design objective for the best Gabor filter is based on the minimization principle that finds the minimum output response of a homogeneous texture pattern in the training process. By defining a non-negative output response, each homogeneous texture region defined in a sliding window will have output amplitude close to zero, and any untrained defect region will have significantly high output amplitude. A pixel with zero response can be logically considered as a fine particle, and a pixel with large response is a coarse particle. Therefore, all pixels associated with the homogeneous texture will be "sifted" out, and only those pixels corresponding to local inhomogeneity will remain on the "screen". The statistical process control principle is then used to set up the control limits of output amplitude for distinguishing between defective regions and homogeneous regions in the filtered image.

This paper is organized as follows: section 2 describes the Gabor filtering scheme, defines the output response of a filtered image, and discusses the design of the best Gabor filter. Section 3 demonstrates the experimental results for a variety of 
real textured-surfaces including textile fabric, machined surface, leather, sandpaper, and natural wood. This paper is concluded in Section 4.

\section{DISCRIMINATING FILTER DESIGN}

We begin with a brief overview of Gabor filters. The 1-D Gabor function was first defined by Gabor [30], and later extended to 2-D by Daugman [21]. A 2-D Gabor filter is an oriented complex sinusoidal grating modulated by a 2-D Gaussian function, which is given by

$$
G_{\sigma, \phi, \theta}(x, y)=g_{\sigma}(x, y) \cdot \exp [2 \pi j \phi(x \cos \theta+y \sin \theta)]
$$

where

$$
g_{\sigma}=\frac{1}{2 \pi \sigma^{2}} \exp \left[-\left(x^{2}+y^{3}\right) / 2 \sigma^{2}\right], \text { and } j=\sqrt{-1}
$$

The frequency of the span-limited sinusoidal grating is given by $\phi$ and its orientation is specified as $\theta . g_{\sigma}(x, y)$ is the Gaussian function with scale parameter $\sigma$. The parameters of a Gabor filter are therefore given by the frequency $\phi$, the orientation $\theta$ and the scale $\sigma$.

The Gabor filter $G_{\sigma, \phi, \theta}(x, y)$ forms complex valued function. Decomposing $G_{\sigma, \phi, \theta}(x, y)$ into real and imaginary parts gives

$$
G_{\sigma, \phi, \theta}(x, y)=R_{\sigma, \phi, \theta}(x, y)+j I_{\sigma, \phi, \theta}(x, y)
$$

where

$$
\begin{aligned}
& R_{\sigma, \phi, \theta}(x, y)=g_{\sigma}(x, y) \cdot \cos [2 \pi \phi(x \cos \theta+y \sin \theta)] \\
& I_{\sigma, \phi, \theta}(x, y)=g_{\sigma}(x, y) \cdot \sin [2 \pi \phi(x \cos \theta+y \sin \theta)]
\end{aligned}
$$


Gabor-filtered output of an image $f(x, y)$ is obtained by the convolution of the image with the Gabor filter $G_{\sigma, \phi, \theta}(x, y)$, i.e.

$$
\int_{-\infty}^{\infty} \int_{-\infty}^{\infty} f(x, y) \cdot G_{\sigma, \phi, \theta}(x, y) d x d y
$$

Given a neighborhood window of size $W \times W$ for $W=2 k+1$, the discrete convolutions of $f(x, y)$ with respective real and imaginary components of $G_{\sigma, \phi, \theta}(x, y)$ are

$$
G_{R}(x, y \mid \sigma, \phi, \theta)=\sum_{\mathrm{I}=-k}^{k} \sum_{m=-k}^{k} f(x+\mathrm{I}, y+m) \cdot R_{\sigma, \phi, \theta}(\mathrm{I}, m)
$$

and

$$
G_{I}(x, y \mid \sigma, \phi, \theta)=\sum_{\mathrm{I}=-k}^{k} \sum_{m=-k}^{k} f(x+\mathrm{I}, y+m) \cdot I_{\sigma, \phi, \theta}(\mathrm{I}, m)
$$

Define the energy $E(x, y \mid \sigma, \phi, \theta)$ at $(x, y)$ within the window $W \times W$ as

$$
E(x, y \mid \sigma, \phi, \theta)=C_{R}^{2}(x, y \mid \sigma, \phi, \theta)+C_{I}^{2}(x, y \mid \sigma, \phi, \theta)
$$

Note that the energy defined in eq. (5) is a non-negative real number. If the Gabor-filter parameters are selected so that the corresponding energy is a minimum for a specific texture sample, every filtered subimage that has similar texture pattern as the training sample will generate the energy value close to zero. Any subimage with the texture pattern different from the training one will yield significantly large energy value. This converts the difficult defect detection problem in complicated textured-surfaces into a simple binary thresholding problem where low energy represents homogeneous textures and high energy represents local anomalies. 
In this research, we are considering a supervised inspection problem, i.e., representative samples of the textures of interest are given to help in designing the most discriminating filter. Supervised systems are most appropriate for controlled circumstances in industry. The training sample can be arbitrarily selected from a non-defective region of textured surface. The neighborhood window $W \times W$ is selected so that the representation of self-similarity of a homogeneous texture pattern is sufficient. The self-similarity means that all sufficiently large subimages of a textured image are considered similar independently of their position. For a given training texture $T_{0}$ with size of $W \times W$, the optimal Gabor-filter parameters $(\sigma, \phi, \theta)$ are given by

$$
\min E\left(T_{0} \mid \sigma, \phi, \theta\right)
$$

Subject to

$$
\begin{aligned}
& \sigma_{\text {min }} \leq \sigma \leq \sigma_{\text {max }} \\
& \phi_{\text {min }} \leq \phi \leq \phi_{\text {max }} \\
& 0 \leq \theta \leq 180
\end{aligned}
$$

where $E\left(T_{0} \mid \sigma, \phi, \theta\right)$ is the energy of image $T_{0}$ centered at $(0,0)$, and it can be obtained from eq. (5). The constraints (6.a), (6.b) and (6.c) specify the possible ranges of filter parameters $\sigma, \phi$ and $\theta$, respectively. $\sigma_{\min }$ and $\sigma_{\max }$ are the minimum and maximum values of $\sigma$. Dunn et al. [31] have proposed a guideline on selecting values for the scale parameter $\sigma$. A good practice in implementation is to select $\sigma$ around 10. $\phi_{\min }$ and $\phi_{\max }$ give the minimum and maximum values of the frequency parameter $\phi$. We can generally select $\phi_{\min }=1$ and $\phi_{\max }=W \quad$ (the width of the neighborhood window). We need only to consider the orientation parameter $\theta$ in the interval between $0^{\circ}$ and $180^{\circ}$. Symmetry makes the other 
directions redundant.

The formulated model above is a nonliear constrained programming problem with multiple continuous variables. This may call for sophisticated optimization techniques such as simulated annealing algorithms $[32,33,34]$ to determine the best parameter values of $\sigma, \phi$ and $\theta$. However, empirical study has shown that the energy function defined in eq. (5) is not very sensitive to the small variation of the parameter values. We can consider only integer values, i.e., the resolution of 1 , for each parameter, and still find a best parameter set that makes the energy function converge to zero. Since the training process can be completed off-line, a simple exhaustive search will serve the purpose to find a best parameter set $\left(\sigma^{*}, \phi^{*}, \theta^{*}\right)$ that gives $E\left(T_{0} \mid \sigma^{*}, \phi^{*}, \theta^{*}\right)=0$.

In the inspection process, the selected Gabor filter will slide over the entire sensed image in a pixel-by-pixel basis so that the corresponding energy of every pixel in the image can be determined. The filter will give a minimum response close to zero when the sliding window covers a homogeneous texture region in the image, and will generate large response for an inhomogeneous region. This transforms texture differences into detectable filter output.

A simple statistical process control principle follows to set up the control limits for distinguishing defects from homogeneous textures in the filtered image. Since the desired energy value in the training process is zero, only the upper control limit is required to detect energy deviation in the filtered image. It is given by

$$
\mu_{E}+C \cdot \sigma_{E}
$$


where $C$ is a control constant; $\mu_{E}$ and $\sigma_{E}$ are the mean and standard deviation of energy values in the filtered image of size $N \times M$, i.e.,

$$
\begin{aligned}
\mu_{E} & =\frac{1}{N \times M} \sum_{y=0}^{M-1} \sum_{x=0}^{N-1} E\left(x, y \mid \sigma^{*}, \phi^{*}, \theta^{*}\right) \\
\sigma_{E} & =\left\{\frac{1}{N \times M-1} \sum_{y=0}^{M-1} \sum_{x=0}^{N-1}\left[E\left(x, y \mid \sigma^{*}, \phi^{*}, \theta^{*}\right)-\mu_{E}\right]^{2}\right\}^{1 / 2}
\end{aligned}
$$

$\mu_{E}$ and $\sigma_{E}$ can be pre-computed from the filtered image of a defect-free model. If the energy value of a pixel in the sensed image is smaller than the control limit (threshold) $\mu_{E}+C \cdot \sigma_{E}$, the pixel is classified as a homogeneous element. Otherwise, it is classified as a defective element.

\section{EXPERIMENTS AND DISCUSSION}

\subsection{Experimental results}

In this section we present the experimental results for evaluating the validity of the proposed Gabor-filter approach for textured-surface inspection. All experiments are implemented on a personal computer using the $\mathrm{C}$ language. The algorithm is tested on a number of real textured-surfaces including textile fabric, milled surface, leather, sandpaper and wood. All input images are $512 \times 480$ pixels wide with 8 -bit gray levels. Only the inner $400 \times 400$ pixels are used as the effective image region so that the filter will not extend outside the image boundaries during convolution. The size of the neighborhood window is selected to be $65 \times 65$ pixels for all test samples. In the training process, a subimage of size $65 \times 65$ for each 
textured-surface model (the squared frame as shown in Figure 1(a)) is arbitrarily selected to determine the best filter parameters.

Figure 1(a) shows a textile fabric with the shedding defect on the surface. Figure 1(b) visually shows the output energy as an intensity function, where brightness is proportional to the magnitude of energy. Figure 1(c) presents the plot of the energy function in 3-D perspective. It can be seen from Figures 1(b) and 1(c) that all pixels in the homogeneous texture region have small energy values close to zero, and pixels in the defective region have relatively large energy values. Figure 2(a) shows a milled surface with a scratch defect. Figures 2(b) and 2(c) illustrate the corresponding detection results. Figure 3(a) demonstrates a leather surface with wear. The detection of the wear defect is shown as an intensity function in Figure 3(b), and a 3-D plot in Figure 3(c). Figure 4(a) shows a sandpaper surface. It can be observed from Figures 4(b) and 4(c) that the proposed Gabor-filter detector can easily identify the scratch even though it is a subtle defect on the surface. Table 1 summarizes the selected filter parameter values and the resulting energy values for the training samples shown in Figures 1(a), 2(a), 3(a) and 4(a). Note that all trained energy values are approximately equal to zero.

Table 1 . The trained energy values and filter parameter values.

\begin{tabular}{|l|c|c|}
\hline \multicolumn{1}{|c|}{ Textured image } & $\begin{array}{c}\text { Energy } \\
E\end{array}$ & $\begin{array}{c}\text { Filter parameters } \\
(\sigma, \phi, \theta)\end{array}$ \\
\hline Textile fabric (Fig. 1) & 0.000000 & $(12,62,180)$ \\
\hline Milled surface (Fig. 2) & 0.000000 & $(12,60,61)$ \\
\hline Leather (Fig. 3) & 0.000001 & $(12,45,98)$ \\
\hline Sandpaper (Fig. 4) & 0.000000 & $(11,53,168)$ \\
\hline
\end{tabular}


In the proposed method, there are two major parameters, the neighborhood window $W \times W$ and the control constant $\mathrm{C}$ for the control limit, which may affect the inspection results. We have also conducted experiments to evaluate the impact of varying $W$ and $C$ on the effectiveness of defect inspection.

\subsection{Effect of neighborhood window $W$}

The choice of a proper neighborhood window size must be large enough to contain the local, periodic, spatial arrangement of intensity for the homogeneous texture in question. Too small a window size causes insufficient representation of texture information, whereas too large a window size increases the computational burden. In this experiment, we vary the window size from $65 \times 65,53 \times 53,41 \times 41$ to $25 \times 25$ pixels to study the impact of window size on the output response of energy. Figure 5(a) presents a textile fabric with the shedding defect on the surface. Figures 5(b), 5(c), 5(d) and 5(e) show the detection results as an intensity function from window sizes $65 \times 65,53 \times 53,41 \times 41$ and $25 \times 25$, respectively. Figure 6 (a) shows one additional example of a natural wood surface. The corresponding detection results from the four different window sizes are presented in Figures 6(b), 6(c), 6(d) and 6(e).

Window sizes $65 \times 65$ and $53 \times 53$ generate similar energy representation for the defects. The high-energy regions associated with the defects are significant and highly concentrated. However, as the window sizes are reduced to $41 \times 41$ and $25 \times$ 25 , the number of high-energy pixels in the defective regions becomes small and distributes in a scattering manner. Based on the detection results in Figures 5 and 6 , 
it reveals that an oversized window may not generate better detection, but an undersized window may overlook subtle defects. In actual implementation, the choice of a window size in the range between 50 and 60 pixels is suggested for the trade-off between effectiveness and efficiency.

\subsection{Effect of control constant $C$}

The control limit is employed to distinguish between homogeneous texture regions and defective regions in a filtered image. The upper control limit of energy is placed at a distance $C \cdot \sigma_{E}$ from the mean $\mu_{E}$. Too small the constant value $C$ give tight control and may result in false rejection (type I error). Too large the constant value $C$ gives loose control and may generate false acceptance (type II error).

For the purpose of visual display, pixels in homogeneous texture regions (under the control limit) and pixels in defective regions (above the control limit) are represented, respectively, by black and white intensities as a binary image. Figures 7(a) and 8(a) show the textured surfaces of a textile fabric and sandpaper, respectively. Figures 7(b) and 8(b) present the thresholding results from the control constant $C=3.5$, and Figures 7(c) and 8(c) are the results from the control constant $C=6.0$. In these test samples, a small control constant will result in minor noise in the binarized image. Based on the observation in Figures 1(c), 2(c), 3(c) and 4(c), the energy magnitude of defective elements is significantly larger than that of homogeneous elements. Therefore, a large control constant will generally eliminate noise for both defective and defect-free surfaces. The discrimination between defect 
and noise is not sensitive to the value of $C$ since the random noise is generally very small in size, compared to the area of a defect.

\section{CONCLUSIONS}

In automated surface inspection, one often has to solve the problem of detecting small defects which appear as local anomalies embedded in a homogeneous texture. Traditional local approaches for automated surface inspection compute a set of textural features in a sliding window, and search for significant local deviations in the feature values between the sensed image and the model image. Most inspection techniques that rely on high-level textural features suffer from the difficulty and time-consuming nature of extracting features from each specific textured-surface.

In this paper we have presented a Gabor-filter approach for automatic inspection of defects in both structural and statistical textures. The proposed method does not depend on the extraction of textural features. It is based on the output response of energy from the convolution of the textured image with a specific Gabor filter. The best parameters of a Gabor filter for a given textured-surface is selected so that the energy of the homogeneous texture is zero. Any unpredictable defect patterns in the sensed image will generate significantly large energy values and, therefore, a simple control limit (threshold) can be selected to discriminate between homogeneous elements and defective elements. The experiments on structural textures such as textile fabric and milled surface and statistical textures such as leather and sandpaper have shown the effectiveness of the proposed method to detect non-quantitatively measurable defects. 


\section{REFERENCES}

1. A. Pikaz and A. Averbuch, "An efficient topological characterization of gray-levels textures using a multiresolution representation," Graphical Models and Image Processing, 59, pp. 1-17, 1997.

2. T. S. Newan and A. K. Jain, "A survey of automated visual inspection," Computer Vision and Image Understanding, 61, pp. 231-262, 1995.

3. J. Wilder, "Finding and evaluating defects in glass," in Machine Vision for Inspection and Measurement (Ed. H. Freeman), Academic Press, New York, pp. 237-255, 1989.

4. J. Olsson and S. Gruber, "Web process inspection using neural classification of scattering light," Proceedings of the IEEE International Conference on Industrial Electronics, Control, Instrumentation and Automation (IECON'92), San Diego pp. 1443-1448, 1992.

5. C. Fernandez, C. Platero, P. Campoy and R. Aracil, "Vision system for on-line surface inspection in aluminum casting process," Proceedings of the IEEE International Conference on Industrial Electronics, Control, Instrumentation and Automation (IECON'93), Maui, HA, pp. 1854-1859, 1993.

6. D. Brzakovic and N. Vujovic, "Designing defect classification system: a case study," Pattern Recognition, 29, pp. 1401-1419, 1996.

7. A. Noble, V. D. Nguyen, C. Marinos, A. T. Tran, J. Farley, K. Hedengren and J. L. Mundy, "Template guided visual inspection," Proceedings of the Second European Conference on Compute Vision, Santa Margherita Ligure, Italy, pp. 893-901, 1992.

8. M. Moganti and F. Ercal, "Automatic PCB inspection Algorithms: a survey," Computer Vision and Image Understanding, 63, pp. 287-313, 1996.

9. R. C. Gonzalez and R. E. Woods, Digital Image Processing, Addison-Wesley, Reading, Mass, 1992.

10. F. S. Cohen, "Maximum likelihood unsupervised textured image segmentation," CVGIP : Graphical Models and Image Processing, 54, pp. 239-251, 1992. 
11. M. M. Van Hulle and T. Tollenaere, "A modular artificial neural network for texture processing," Neural Networks, 6, pp. 7-32, 1993.

12. K. V. Ramana and B. Ramamoorthy, "Statistical methods to compare the texture features of machined surfaces," Pattern Recognition, 29, pp. 1447-1459, 1996.

13. R. M. Haralick, K. Shanmugam and I. Dinstein, "Textural features for image classification,” IEEE Trans. System, Man Cybernet., 3, pp. 610-621, 1973.

14. L. H. Siew and R. M. Hogdson, "Texture measures for carpet wear assessment," IEEE Trans. Pattern Anal. Mach. Intell., 10, pp. 92-105, 1988.

15. T. Ojala, M. Pietikäinen and O. Silven, "Edge-based texture measures for surface inspection," Proceedings of the 11th International Conference on Pattern Recognition, The Hague, The Netherlands, pp. B-594 - B-598, 1992.

16. R. W. Conners, C. W. McMillin, K. Lin and R. E. Vasquez-Espinosa, "Identifying and locating surface detects in wood," IEEE Trans. Pattern Anal. Mach. Intell., PAMI-5, pp. 573-583, 1983.

17. H. Wechsler, "Texture analysis: a survey," Signal Processing, 2, pp. 271-282, 1980.

18. S. -S. Liu and M. E. Jernigan, "Texture analysis and discrimination in additive noise," Computer Vision, Graphics, Image Process., 49, pp. 52-67, 1990.

19. D. -M. Tsai, J. -J. Chen and J. -F. Chen, "A vision system for surface roughness assessment using neural networks," International Journal of Advanced Manufacturing Technology, 14, pp. 412-422, 1998.

20. M. Porat and Y. Y. Zeevi, "The generalized Gabor scheme of image representation in biological and machine vision," IEEE Trans. Pattern Anal. Mach. Intell., 10, pp. 452-468, 1988.

21. J. G. Daugman, "Uncertainty relation for resolution in space, spatial-frequency, and orientation optimized by two-dimensional visual cortical filters," J. Opt. Soc. Amer., 2, pp. 1160-1169, 1985. 
22. R. L. De Valois, D. G. Albrecht and L. G. Thorell, "Spatial-frequency selectivity of cells in macaque visual cortex," Vision Research, 22, pp. 545-559, 1982.

23. J. Beck, A. Sutter and R. Ivry, "Spatial frequency channels and perceptual grouping in texture segregation," Comput. Vision, Graphics, Image Processing, 37, pp. 299-325, 1987.

24. M. Clark and A. C. Bovik, "Texture segmentation using Gabor modulation/ demodulation," Pattern Recognition Letters, 6, pp. 261-267, 1987.

25. D. Dunn and W. E. Higgins, "Optimal Gabor filters for texture segmentation," IEEE Trans. Image Processing, 4, pp. 947-964, 1995.

26. A. Teuner, O. Pichler and B. J. Hosticka, "Unsupervised texture segmentation of images using tuned matched Gabor filters," IEEE Trans. Image Processing, 4, pp. 863-870, 1995.

27. T. P. Weldon, W. E. Higgins and D. F. Dunn, "Efficient Gabor filter design for texture segmentation,” Pattern Recognition, 29, pp. 2005-2015, 1996.

28. T. P. Weldon, W. E. Higgins and D. F. Dunn, "Gabor filter design for multiple texture segmentation,” Optical Engineering, 35, pp. 2852-2863, 1996.

29. P. P. Raghu and B. Yegnanarayana, "Segmentation of Gabor-filtered textures using deterministic relaxation," IEEE Trans. Image Processing, 5, pp. 1625-1636, 1996.

30. D. Gabor, "Theory of communication," J. Inst. Elec. Eng., 93, pp. 429-457, 1946.

31. D. Dunn, W. Higgins and J. Wakeley, "Texture segmentation using 2-D Gabor elementary function," IEEE Trans. Pattern Anal. Mach. Intell., 16, pp. 130-149, 1994.

32. S. Kirkpatrick, C. D. Gelatt, Jr. and M. P. Vecchi, "Optimization by simulated annealing," Science, 220, pp. 671-680, 1983. 
33. R. A. Rutenbar, "Simulated annealing algorithm: an overview," IEEE Circuits and Device Magazine, 5, pp. 19-26, 1989.

34. M. -C. Chen and D. -M. Tsai, "A simulated annealing approach for optimization of multi-pass turning operations," International Journal of Production Research, 34, pp.2803-2825, 1996. 


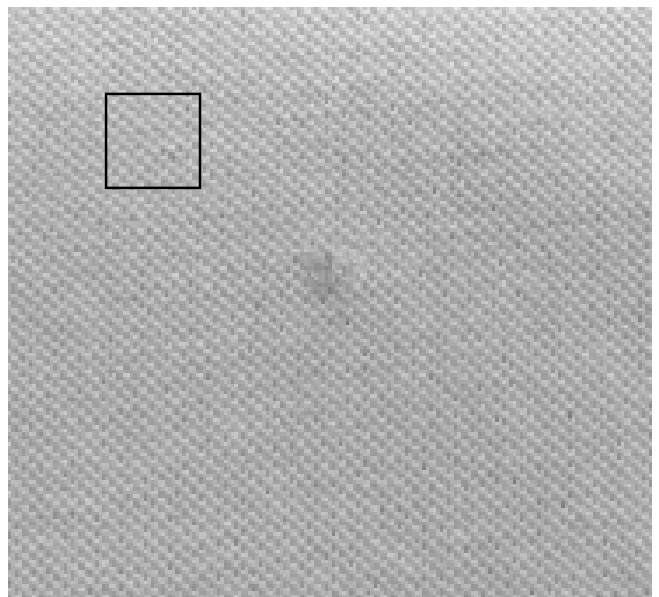

(a)

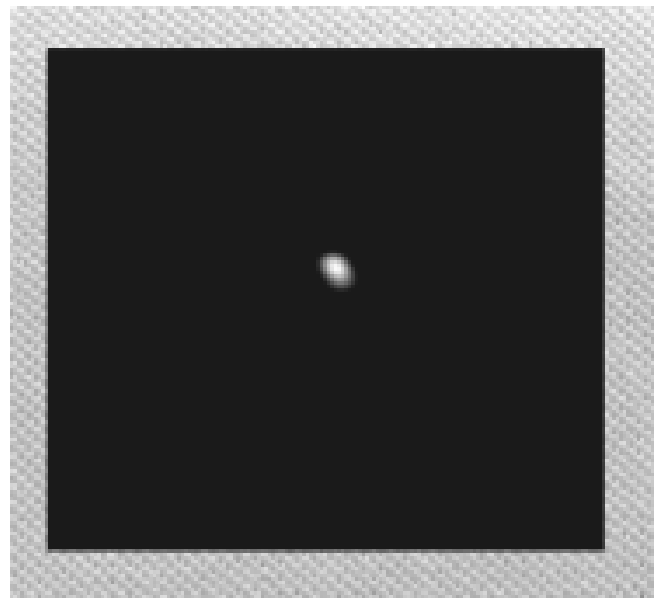

(b)

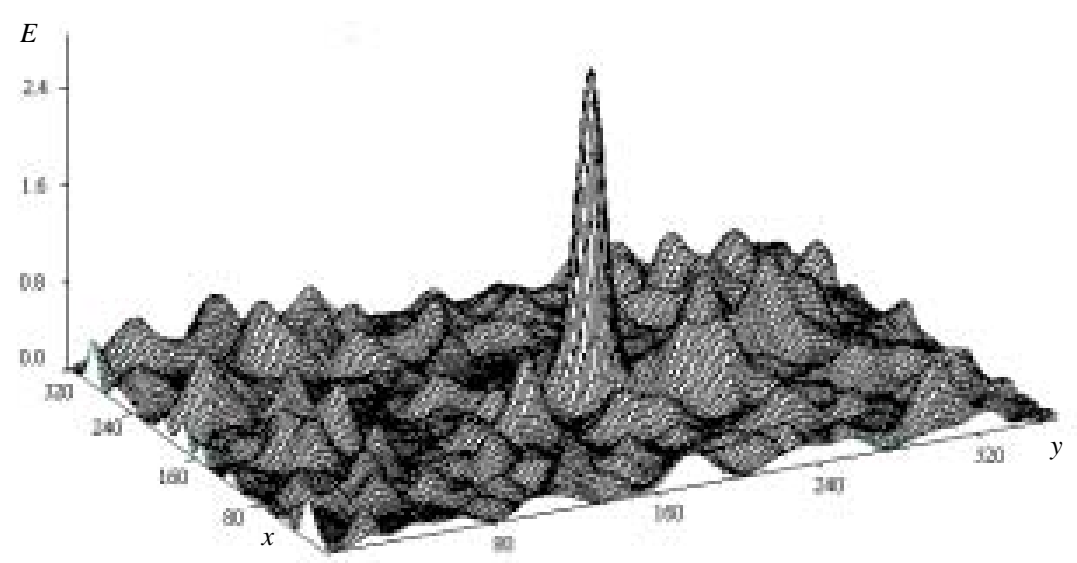

(c)

Figure 1. (a) The original image of a textile fabric, (b) visual display of the energy as an intensity function, (c) the energy function in 3-D perspective. 


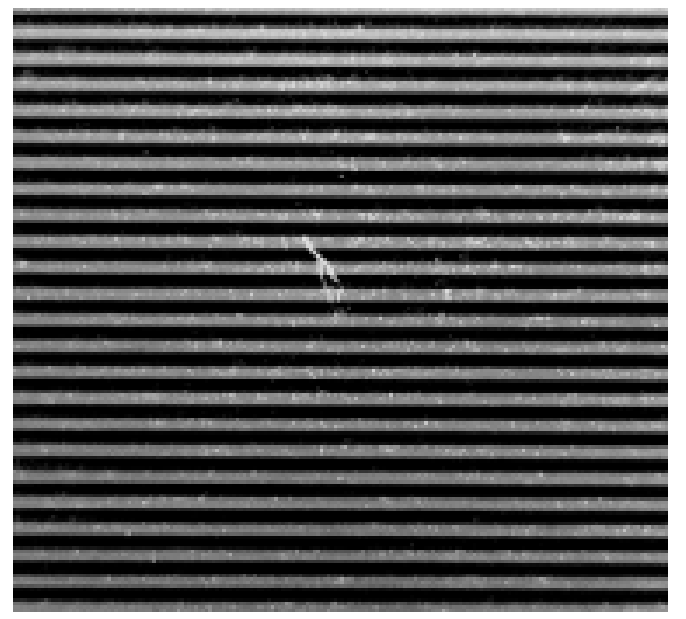

(a)

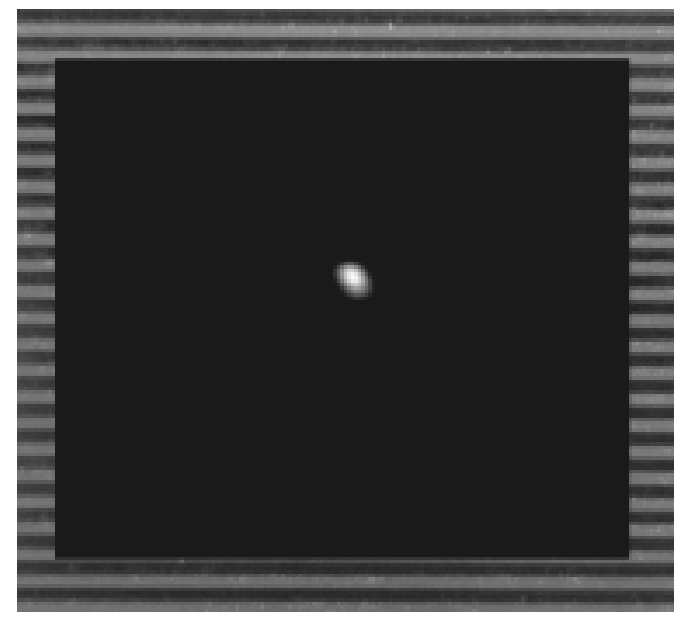

(b)

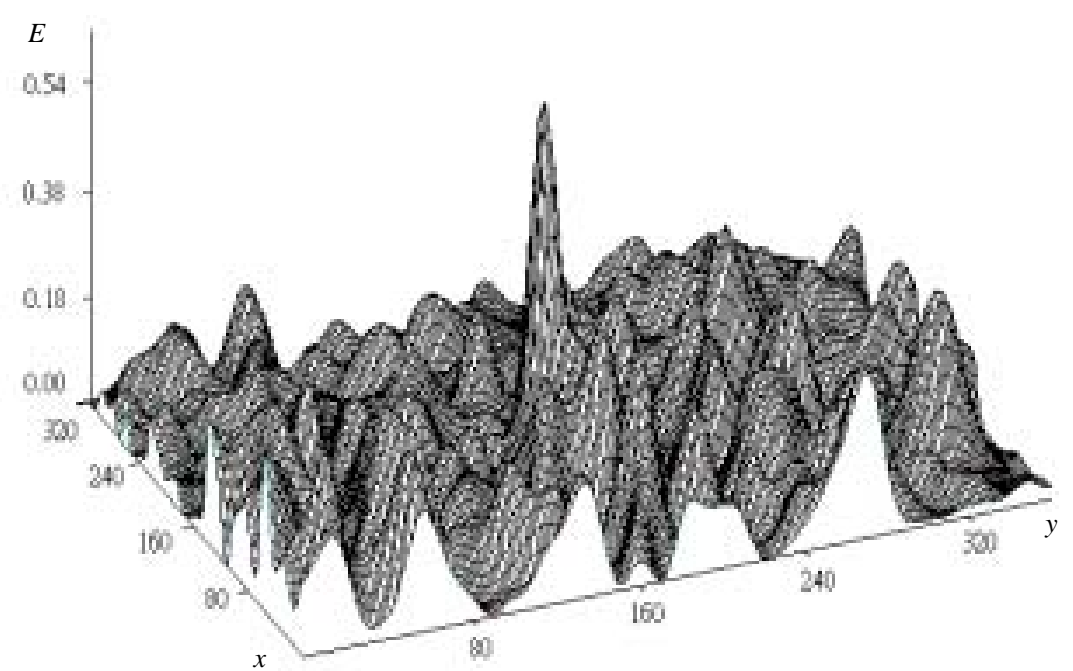

(c)

Figure 2. (a) The original image of a milled surface, (b) visual display of the energy as an intensity function, (c) the energy function in 3-D perspective. 


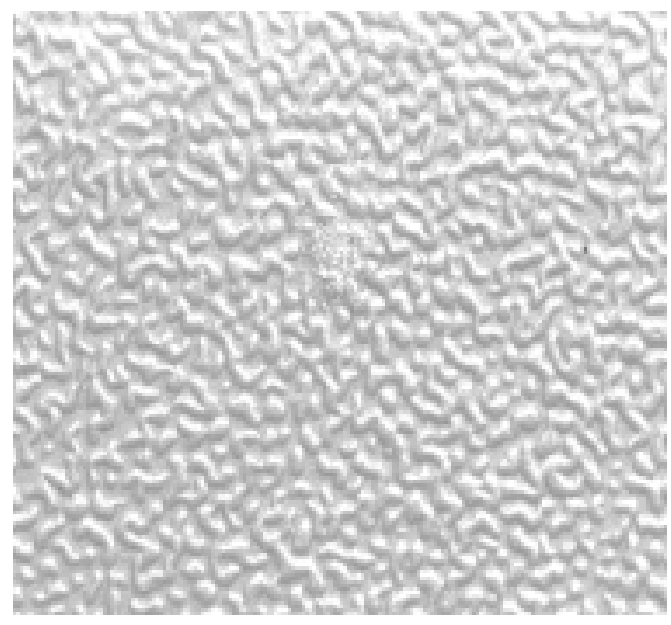

(a)

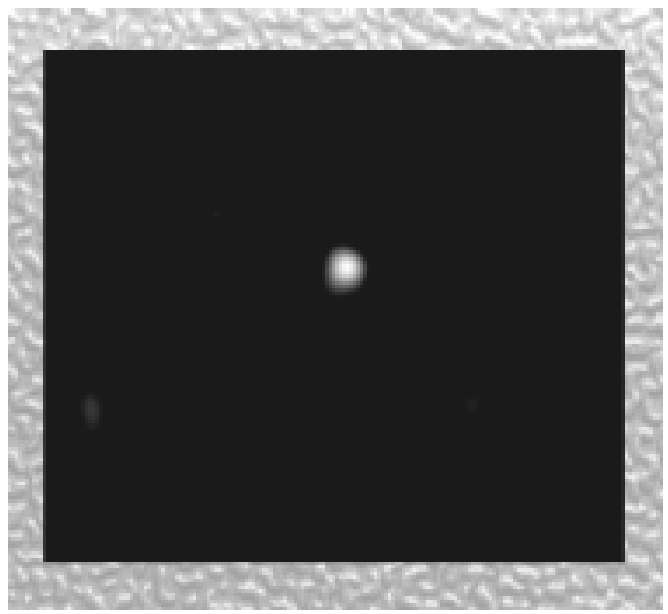

(b)

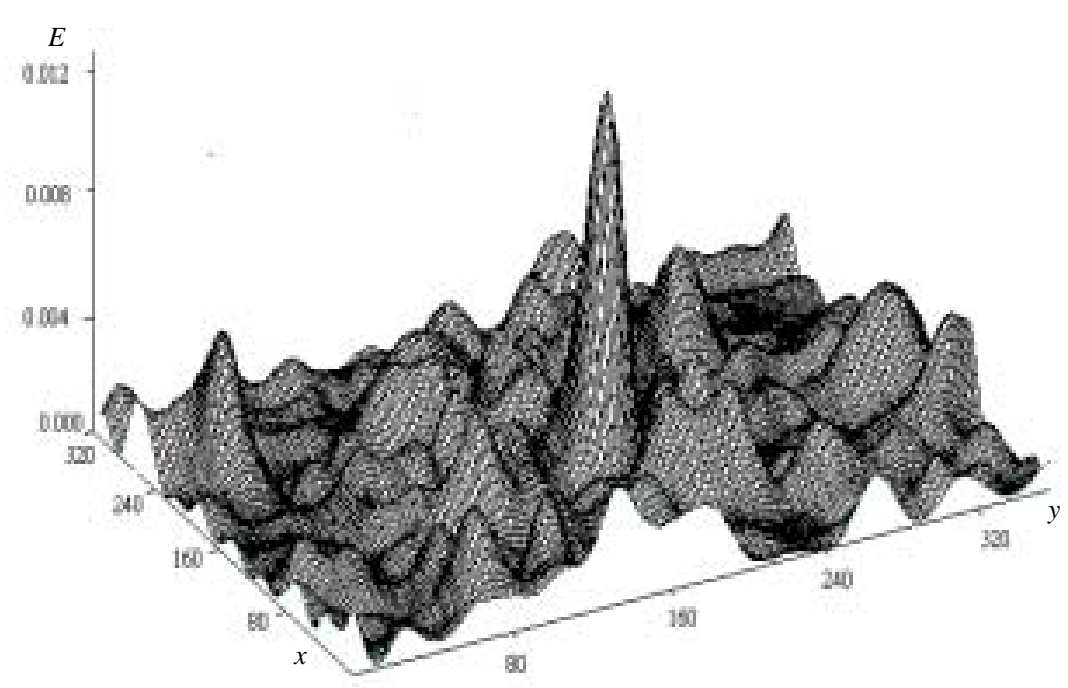

(c)

Figure 3. (a) The original image of a leather surface, (b) visual display of the energy as an intensity function, (c) the energy function in 3-D perspective. 


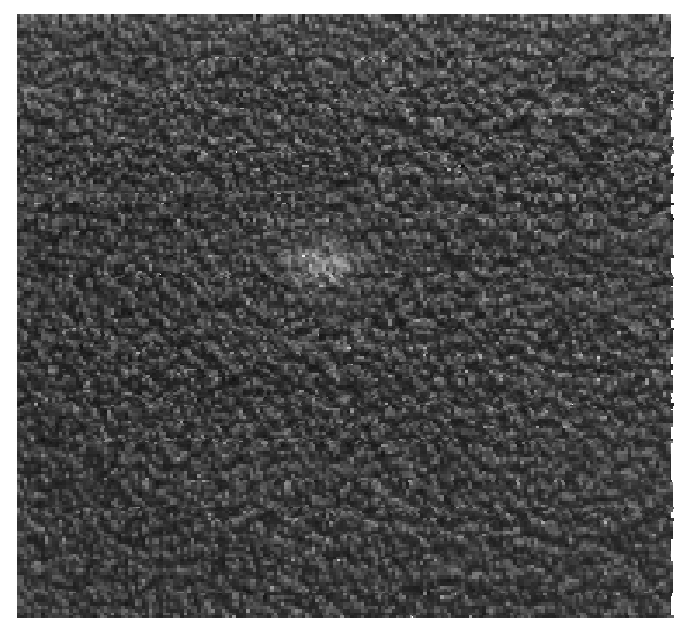

(a)

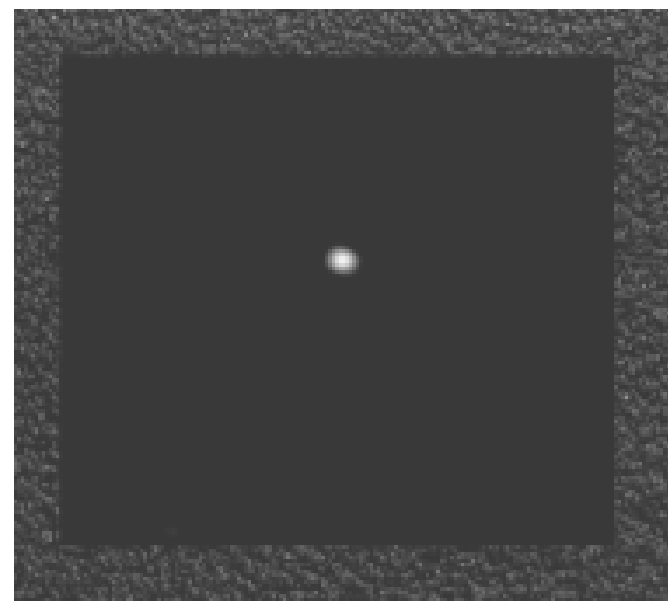

(b)

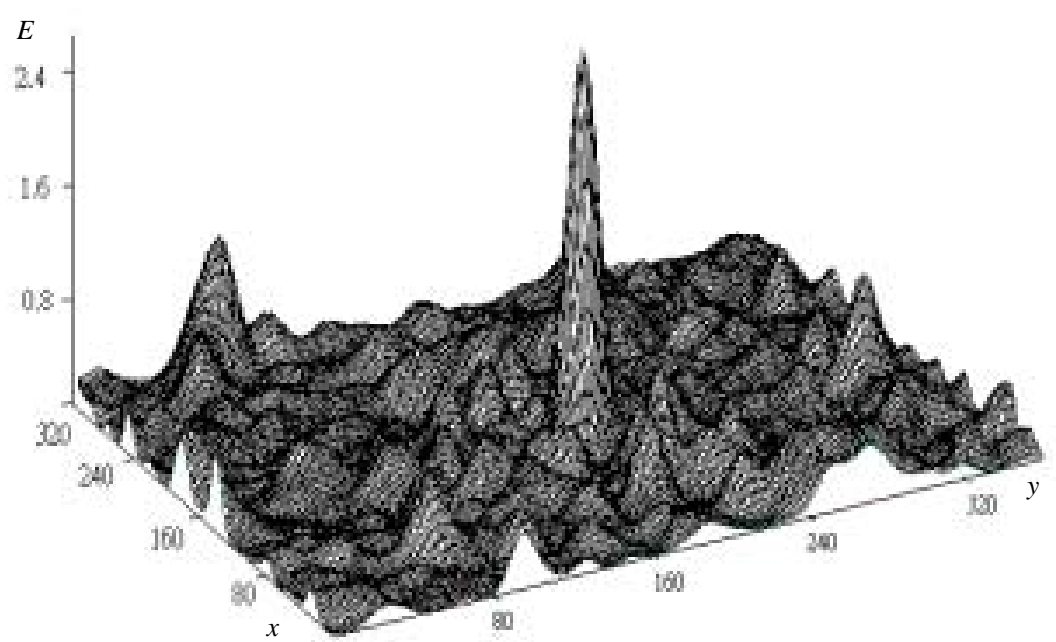

(c)

Figure 4. (a) The original image of a sandpaper surface, (b) visual display of the energy as an intensity function, (c) the energy function in 3-D perspective. 


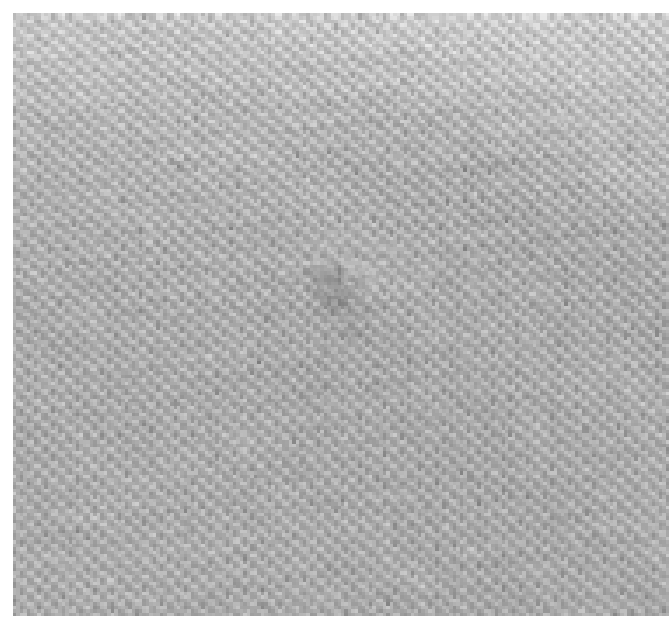

(a)

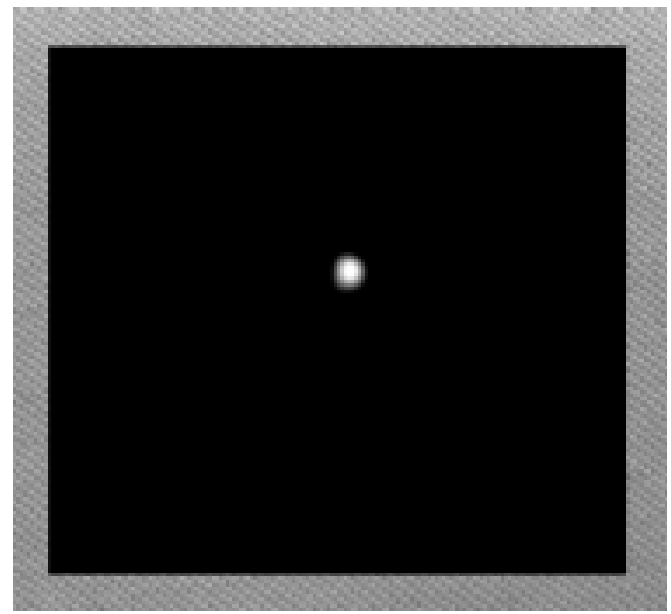

(b) $65 \times 65$

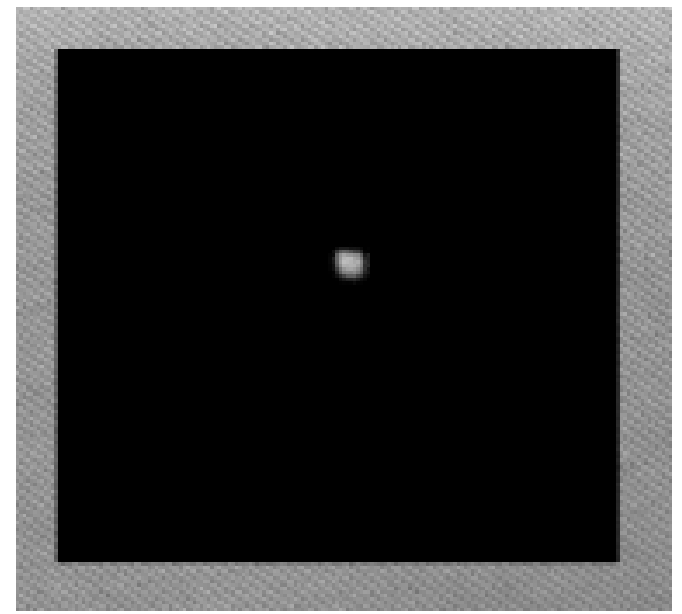

(d) $41 \times 41$

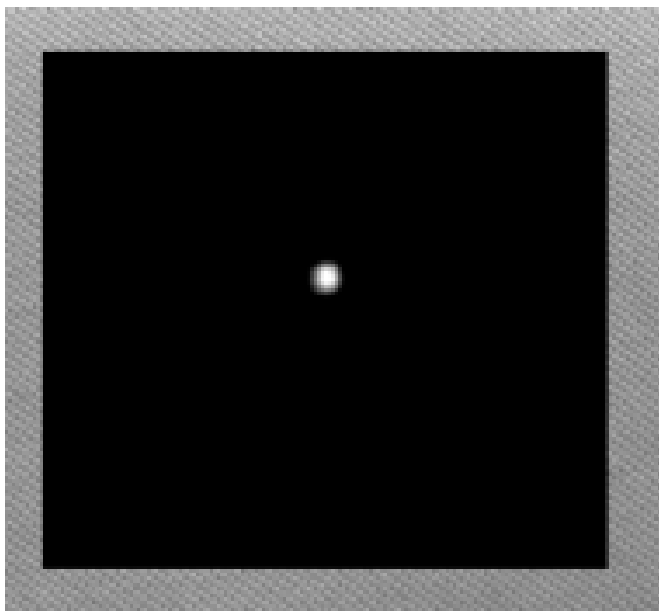

(c) $53 \times 53$

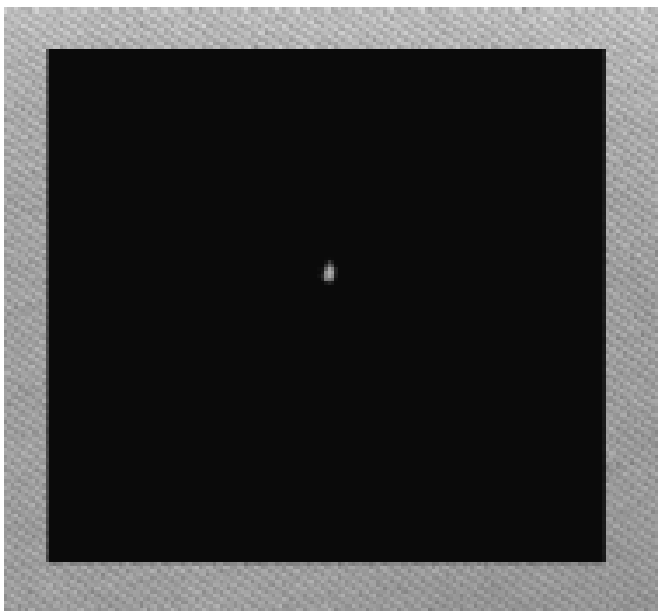

(e) $25 \times 25$

Figure 5. Effect of the window size $W \times W$ : (a) the test sample of a textile fabric, (b) result from window size $65 \times 65$, (c) result from window size $53 \times 53$, (d) result from window size $41 \times 41$, (e) result from window size $25 \times 25$. 


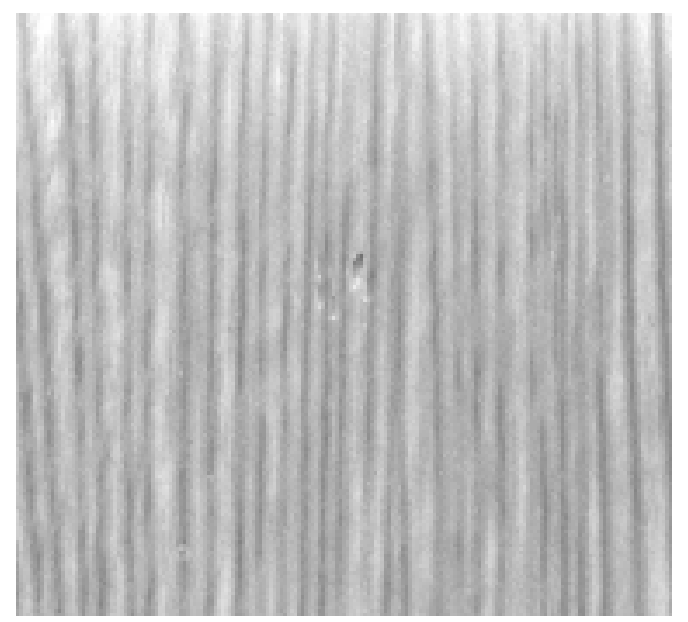

(a)

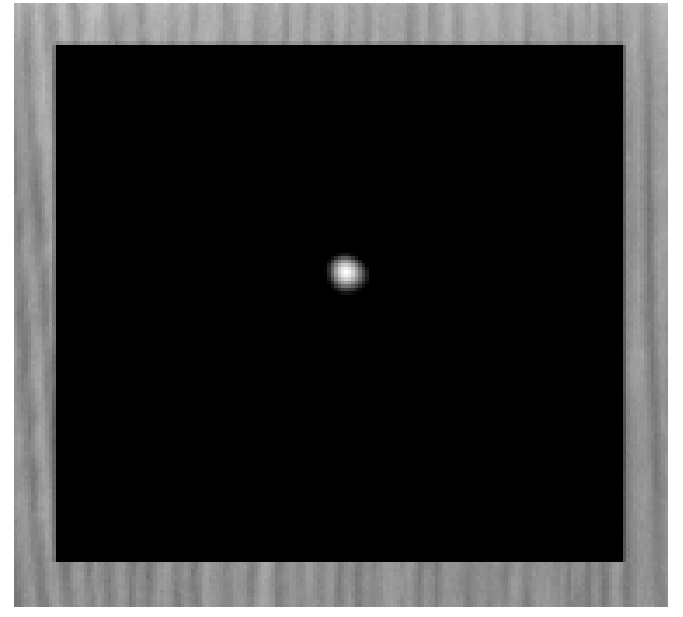

(b) $65 \times 65$

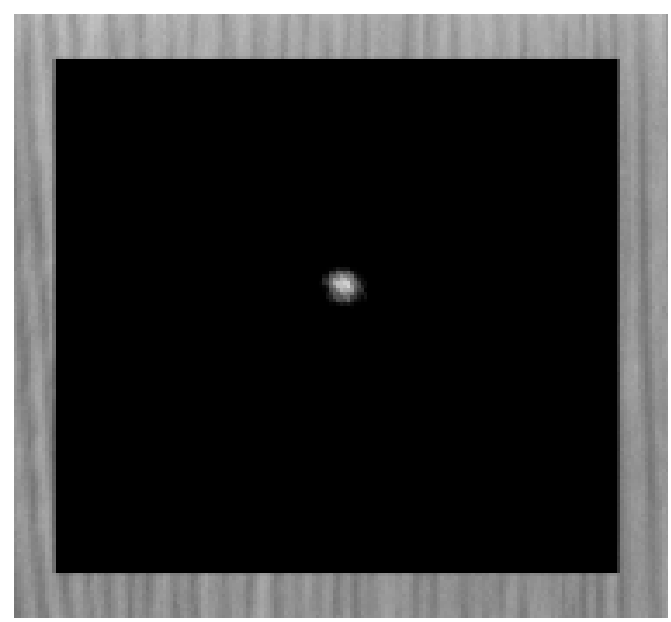

(d) $41 \times 41$

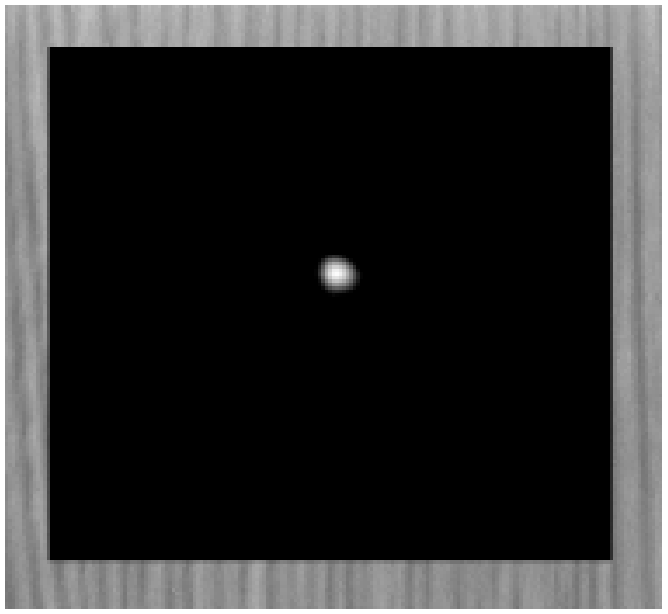

(c) $53 \times 53$

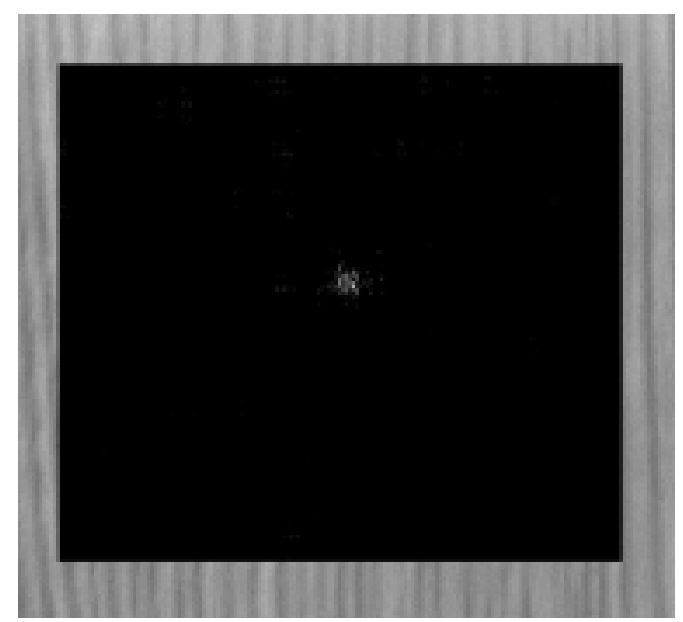

(e) $25 \times 25$

Figure 6. Effect of the window size $W \times W:$ (a) the test sample of a wood surface, (b) result from window size $65 \times 65$, (c) result from window size $53 \times 53$, (d) result from window size $41 \times 41$, (e) result from window size $25 \times 25$. 


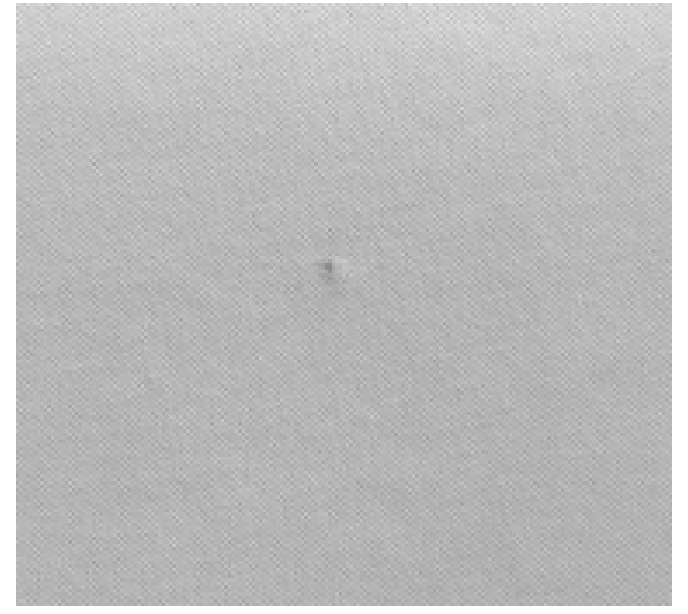

(a)

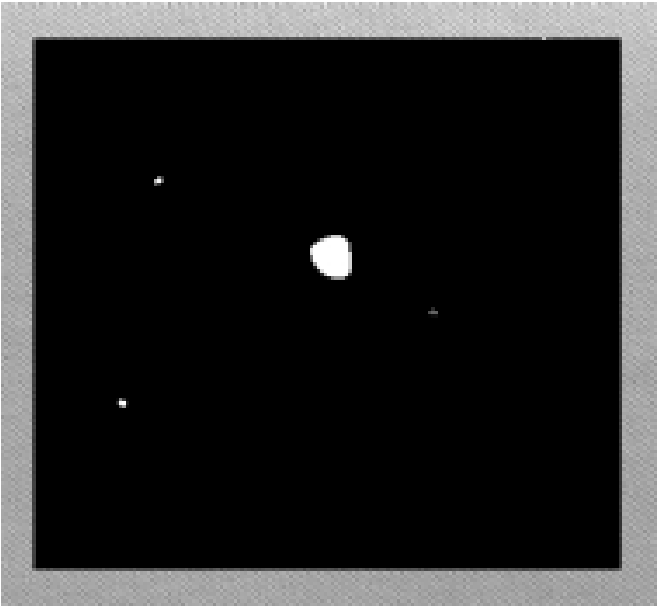

(b) $C=3.5$

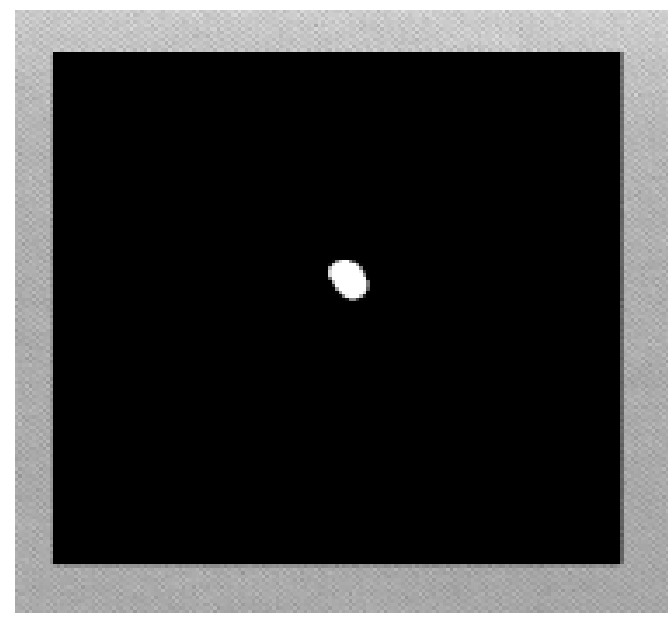

(c) $C=6.0$

Figure 7. Effect of the control constant $C$ : (a) the test sample of a textile fabric, (b) thresholding result from $C=3.5$, (c) thresholding result from $C=6.0$.

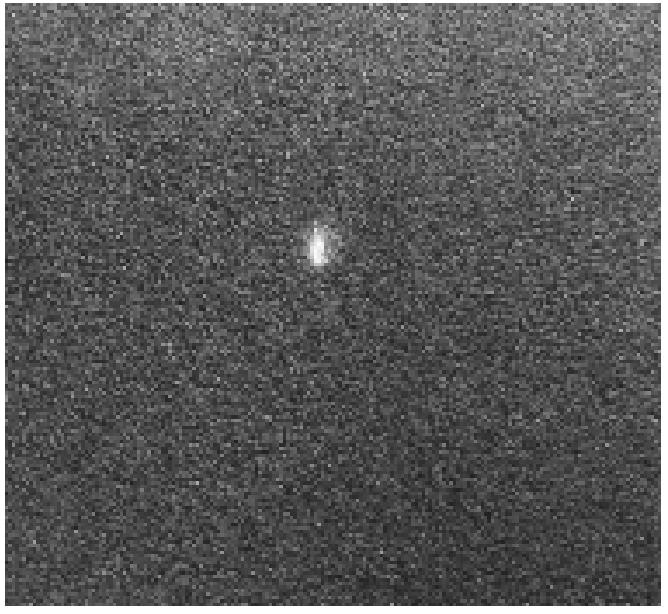

(a)

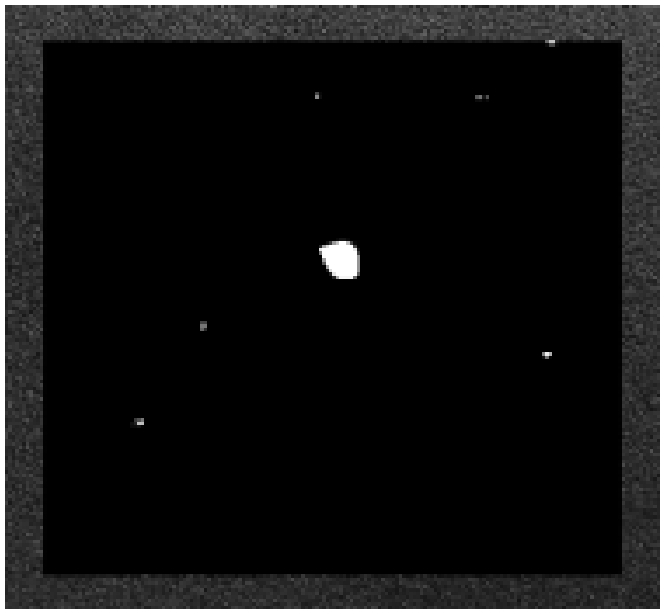

(b) $C=3.5$

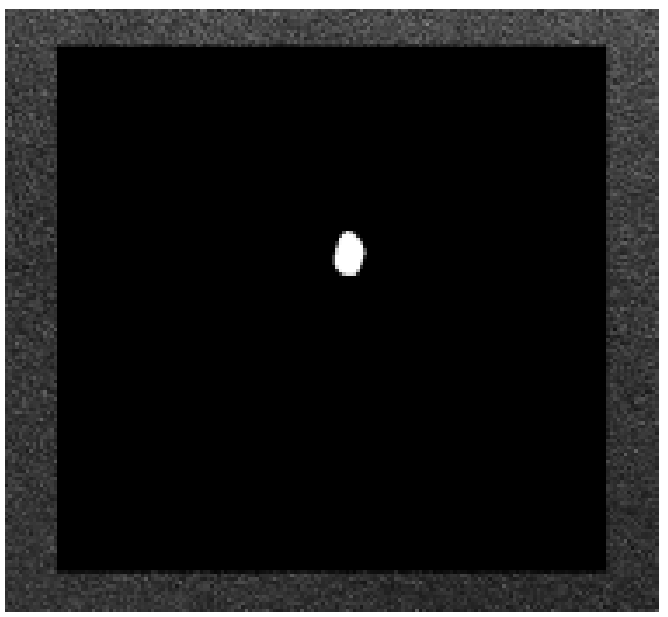

(c) $C=6.0$

Figure 8. Effect of the control constant $C$ :

(a) the test sample of sandpaper,

(b) thresholding result from $C=3.5$,

(c) thresholding result from $C=6.0$. 